\title{
Cierre parcial con sutura subcuticular continua y cicatrización por segunda intención: una técnica útil en cirugía dermatológica
}

Partial closure with purse string suture and healing by secondary intention: A useful technique in dermatologic surgery

\section{Germán Montes ${ }^{1}$, Lucía van den Enden², Consuelo Vélez ${ }^{3}$}

1. Médico, residente de Dermatología, Facultad de Ciencias para la Salud, Universidad de Caldas, Manizales, Colombia

2. Médica dermatóloga; docente, posgrado en Dermatología, Universidad de Caldas, Manizales, Colombia

3. Enfermera epidemióloga, Ph. D. en Salud Pública; docente asociada, Facultad de Ciencias para la Salud, Universidad de Caldas, Manizales, Colombia

\section{Resumen}

La resección de lesiones cutáneas generalmente resulta en defectos ovales o redondos con varias opciones para su reparación. El cierre parcial con sutura subcuticular continua es una técnica que, sumada a la cicatrización por segunda intención, permite excelentes resultados cosméticos y funcionales. La integración de estas dos técnicas le permite al dermatólogo contar con una excelente herramienta de bajo costo, de fácil acceso y con una baja tasa de complicaciones.

Objetivo. Caracterizar y promover el uso de esta técnica quirúrgica en la cirugía dermatológica, resaltando sus ventajas y resultados.

Materiales y métodos. Se llevó a cabo un estudio de serie de casos de lesiones dermatológicas que requerían manejo quirúrgico, cuyas características no permitieron su cierre primario y que reunían características para la aplicación de esta técnica. Se incluyeron pacientes de la consulta del posgrado de Dermatología de la Universidad de Caldas, de julio de 2011 a enero de 2013.

Resultados. Se intervinieron 16 casos con defectos quirúrgicos cerrados mediante sutura subcuticular continua y cicatrización por segunda intención. El aspecto final de la cicatriz se clasificó entre bueno y excelente, y el tamaño de la cicatriz en todos los casos fue menor al del defecto quirúrgico inicial; no se presentaron eventos adversos de importancia.

Conclusión. Aunque en cirugía dermatológica hay una variedad de opciones reconstructivas, en muchos casos, la opción más simple puede brindar los mejores resultados. La combinación de estas dos técnicas se convierte en una herramienta eficaz para el dermatólogo.

PAlabRAS ClAVE: sutura subcuticular continua, bolsa de tabaco, jareta, cicatrización por segunda intención, cirugía dermatológica.

\section{Summary}

Resection of skin lesions usually results in round or oval defects with various options for repair. Partial closure with subcuticular suture is a technique that combined with healing by secondary intention, creates excellent cosmetic and functional results. Integration of these two techniques allows the dermatologist to have an excellent and inexpensive therapeutic tool, easily accessible and with a low complications rate.

\section{Correspondencia:}

Germán Montes

Email:

gamon23@hotmail.com

Recibido: 30 septiembre de 2013. Aceptado: 30 de octubre de 2013.

No se reportan conflictos de intereses. 
Objective: To characterize and promote the use of this surgical technique in dermatological surgery, highlighting its benefits and results.

Materials and methods: A series of cases of skin lesions that required surgical management is presented, whose characteristics did not allow primary closure and features to meet the application of this technique. Patients were evaluated at University of Caldas, between July 2011 and January 2013.

Results: We report 16 cases with surgical defects closed using subcuticular continuous suture and healing by secondary intention. The final appearance of the scar was rated good to excellent, and the size of the scar in all cases was less than the initial surgical defect; there were no significant adverse events.

Conclusion: Although in dermatological surgery there are many reconstructive options in many cases the simplest option can provide the best and most consistent results. The combination of these two techniques becomes an effective tool for dermatologists.

Keywords: Continuous subcuticular suture, snuff bag, purse, healing by second intention, dermatologic surgery.

\section{Introducción}

La extirpación quirúrgica de lesiones cutáneas benignas o malignas frecuentemente resulta en defectos de forma redonda $u$ oval $^{1}$. Usualmente hay cuatro opciones para tratar la herida resultante: cicatrización por segunda intención, cierre primario, rotación de un colgajo o injerto de piel ${ }^{1,2}$.

El cierre de una herida quirúrgica puede ser complejo, dependiendo esencialmente de la localización anatómica y del tamaño de la herida resultante ${ }^{3}$. La forma de la herida y su profundidad, el tipo de tumor resecado, al igual que el estado de salud del paciente y su habilidad para los cuidados posoperatorios, también influyen en la opción de tratamiento escogido ${ }^{4}$.

El cierre primario tiene limitaciones para defectos grandes, especialmente si el tejido tiene pobre laxitud que limite la aproximación de los bordes ${ }^{5}$. El injerto autólogo de piel implica una zona donante, produciendo una nueva herida quirúrgica a distancia, y un colgajo implica una desestructuración anatómica regional ${ }^{3}$.

En los casos de resección de tumores, el riesgo de recurrencia debe influir sobre la opción de reparación o posiblemente retardar la reparación definitiva. Si el riesgo de recurrencia o de tumor residual es considerable, los cierres como los injertos de espesor total y los colgajos, pueden poner en peligro la vigilancia del área y retrasar su diagnóstico. En tal situación, la cicatrización por segunda intención o el injerto de espesor parcial, pueden reducir el riesgo de enmascarar un tumor residual y permiten el seguimiento visual del sitio ${ }^{4}$.
Este proyecto de investigación pretendía caracterizar y promover el uso de esta técnica quirúrgica en el campo de la cirugía dermatológica, resaltando sus ventajas y evidenciando sus excelentes resultados.

\section{Materiales y métodos}

Se llevó a cabo un estudio de serie de casos en el cual se incluyeron pacientes con lesiones dermatológicas que requirieran manejo quirúrgico, cuyas características no permitían el cierre primario de la herida y que reunían las características ideales para la aplicación de esta técnica quirúrgica. Se incluyeron los pacientes evaluados en una de las consultas del posgrado de Dermatología de la Universidad de Caldas, en el período de julio de 2011 a enero de 2013.

Una vez hecha la escisión del la lesión, se utilizó la sutura en bolsa de tabaco, iniciándola y finalizándola externamente a medio centímetro del borde de la herida (FIGURA 1).

A cada paciente se le explicaron los riesgos y beneficios de la técnica, y los cuidados posoperatorios necesarios, que incluían curaciones cada tres días con ungüento antibiótico y su oclusión permanente con gasa con vaselina, hasta el retiro de la sutura en la segunda o la tercera semana. Se diligenció un formato de consentimiento informado, en el que se autorizaba su participación en el estudio y la toma de fotografías clínicas.

Se analizaron edad, sexo, diagnóstico, localización de la lesión, tamaño del defecto quirúrgico antes de la su- 


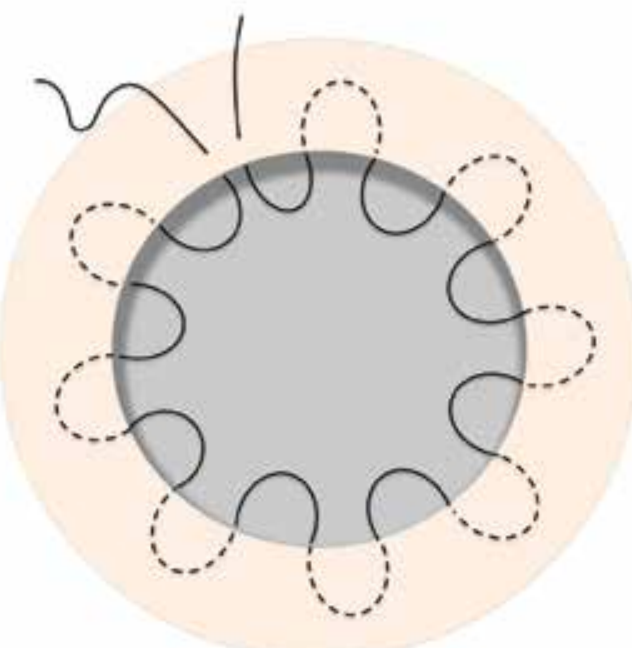

FIGURA 1. Sutura subcuticular que se inicia y se termina en la epidermis.

tura y después de ella, porcentaje de disminución del defecto, complicaciones, sutura utilizada y el nivel de satisfacción del paciente. Se analizaron las diferentes variables estadísticas mediante el SPF 15.00 con licencia para su uso por la Universidad de Caldas.

Este estudio contó con la aprobación del Comité de Bioética de la Facultad de Ciencias para la Salud de la Universidad de Caldas, Manizales.

\section{Resultados}

Se incluyeron 16 pacientes con lesiones dermatológicas que podían tratarse quirúrgicamente, 6 mujeres y 10 hombres, con una edad promedio de $66 \pm 17,7$ años en ambos sexos; el paciente de menor edad tenía 25 años y, el mayor, 93 años.

El $50 \%$ de los casos correspondió a carcinomas basocelulares, $25 \%$ a queratoacantoma, $12,5 \%$ a melanoma, $6,3 \%$ a carcinoma escamocelular y $6,3 \%$ a nevus melanocítico congénito.

En cuanto a la localización, 9 lesiones $(56,3 \%)$ se ubicaban en la región facial, 6 (37,5\%) en el tronco y 1 $(6,3 \%)$ en miembros superiores. Luego de la resección, la forma del defecto quirúrgico resultante fue ovalada en 8 casos $(50 \%)$ y redonda en los restantes $8(50 \%)$.

$\mathrm{El}$ área promedio del defecto quirúrgico inicial (antes del cierre parcial con jareta) fue de $649,43 \pm 562,2 \mathrm{~mm}^{2}$; el defecto de menor tamaño tenía un área de $78,54 \mathrm{~mm}^{2} \mathrm{y}$, el mayor, una de $2.023,19 \mathrm{~mm}^{2}$.
El área promedio del defecto quirúrgico después de la sutura fue de $113,92 \pm 107,1 \mathrm{~mm}^{2}$, con un promedio de disminución del defecto quirúrgico del 81,5\%. En áreas de piel de mayor laxitud se obtuvo un porcentaje de reducción del defecto quirúrgico hasta de 94,83 \% y, en sitios anatómicos de menor laxitud cutánea, hasta de 66,29\%.

En todos los casos se utilizó sutura de monofilamento de tipo polipropileno con diámetros entre 3-0 y 5-0, a criterio del dermatólogo tratante según el tamaño del defecto y la laxitud cutánea del sitio anatómico en tratamiento. Se usó sutura 3-o en 6 pacientes (37,3\%), sutura 4-o en $6(37,3 \%)$ y sutura 5-o en $4(25 \%)$.

En el 87,5\% de los casos (14) no se presentaron eventos adversos, en uno (6,3\%) se confirmó por estudio anatomo-patológico la persistencia tumoral en el margen profundo (queratoacantoma) y en otro $(6,3 \%)$ hubo dehiscencia de sutura después de un esfuerzo físico realizado por el paciente (melanoma en el dorso).

El nivel de satisfacción del paciente con el procedimiento y el resultado final fue calificado como bueno en 10 casos $(62,5 \%)$ y como excelente en 8 casos (37,5\%).

En las FIGURAS 2 Y 3 se presentan dos casos representativos.

\section{Discusión}

La sutura subcuticular continua, bolsa de tabaco o jareta recibe en inglés el nombre de purse string suture, que hace referencia a una bolsa portamonedas y al cordel que la cierra ${ }^{6}$ (FIGURA 4). Su objetivo básico es el reclutamiento de la piel desde todo el diámetro de la lesión para disminuir su tamaño. El defecto residual puede ser reparado con un injerto o dejarse para cicatrización por segunda intención ${ }^{7,8}$.

Fue introducida por primera vez en la reconstrucción facial para reducir la superficie de la herida como un complemento al injerto de piel de espesor total ${ }^{9}$. Su uso original, a principios de la década de 1950, se le atribuye a Bradford Cannon, quien utilizó la técnica después de la extirpación de un quiste sebáceo malar. Él también enseñó este método a los residentes y estudiantes de medicina de Harvard University en el Servicio de Cirugía Plástica del Massachusetts General Hospital ${ }^{10}$.

Peled, et al., fueron los primeros en describir la sutura en bolsa de tabaco en la cirugía reconstructiva de cáncer de cabeza y cuello. En 1992, Brady, et al., presentaron el cierre con sutura en bolsa de tabaco en la literatura dermatológica, como complemento del injerto de piel de espesor total en la reconstrucción facial tras la cirugía micrográfica de Mohs ${ }^{6,7}$. Cuatro años más tarde, Greenbaum y Radonich ampliaron la utilidad de esta técnica mediante la introducción de la idea de que el cierre en bolsa de tabaco puede ser utilizado como un 


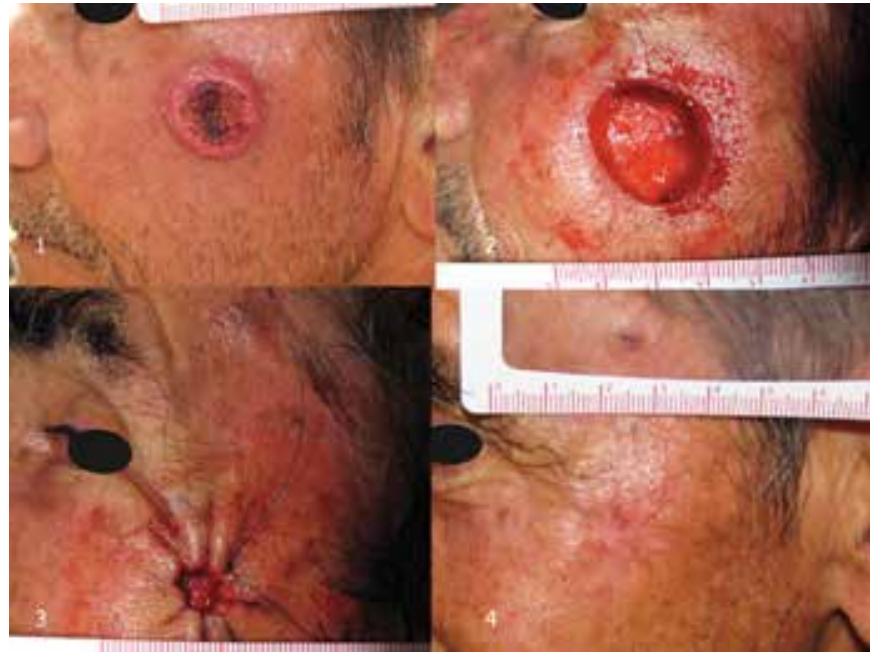

Figura 2. Queratoacantoma; sutura utilizada: polipropileno 4-o A. Lesión clínica; B. Defecto quirúrgico; C. Defecto suturado; D. Aspecto a las 8 semanas.

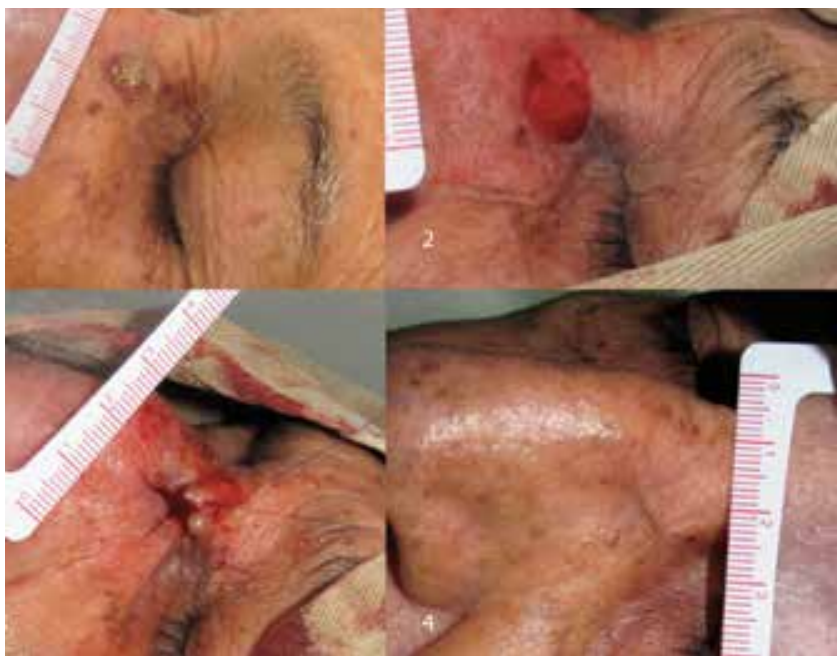

Figura 3. Carcinoma escamocelular; sutura utilizada: polipropileno 5-o. Lesión clínica; B. Defecto quirúrgico; C. Defecto suturado; D. Aspecto a las 10 semanas. procedimiento independiente para el cierre de defectos redondos y ovalados ${ }^{4,5}$.

Se han descrito varios métodos para la colocación de la sutura, como la versión completamente subcuticular y otra que se inicia y finaliza externamente, a medio centímetro del borde de la herida -utilizada en este estudio-. Otra opción es la combinada con bucles de escape ubicados a las 3, 6, 9 y 12 de las posiciones de las agujas reloj, que permiten un retiro más fácil de los puntos ${ }^{4,11}$.

Esta sutura reduce el tamaño de la herida entre 53 y $89 \%$, dependiendo del tamaño ${ }^{12,13}$. Brady, et al., reportaron una reducción promedio del defecto de $53 \%$ $(30-89 \%)^{14}$. En nuestro estudio, el porcentaje promedio de disminución del defecto fue de $81,5 \%$.

El nudo determina el área final que, aunque aparentemente simple, es un componente crucial del resultado con respecto a la contracción del tejido. Este nudo se puede ejecutar fácilmente en tejidos laxos, pero puede ser complejo por la resistencia ofrecida en tejidos más densos ${ }^{12,15}$.

La duración recomendada de la sutura varía. La mayoría de investigadores la retiran después de tres a cuatro semanas $^{11,13}$. En el presente estudio, la sutura se retiró entre la segunda y la tercera semana. Se prefieren suturas no absorbibles y de monofilamento, pues disminuyen el arrastre del tejido, tienen mayor facilidad para anudarse, disminuyen el riesgo teórico de ser nido de infecciones y su remoción es más fácil por ser más res- baladizas $^{13}$. En el presente estudio se utilizó sutura de monofilamento, de tipo polipropileno.

La extensibilidad y la laxitud de la piel son propiedades que permiten el uso de la técnica de sutura en bolsa de tabaco, que inicialmente provoca una considerable distorsión de la piel, y una gradual y sostenida mejoría estética ${ }^{13}$.

Esta sutura es útil cuando se desean resultados cosméticos superiores, puesto que no penetra la epidermis a lo largo de la longitud de la herida. Clásicamente, sólo se perfora la superficie de la piel al principio y al final de la sutura ${ }^{16}$. Se ha utilizado en diferentes especialidades médicas ${ }^{17}$. El principio permanece sin cambios, a pesar de sus diversas aplicaciones, desde mamoplastia reductora y mastopexia dérmica, cateterismos y técnicas endoscópicas, hasta cierre de fístulas uretrales y reparación de hernias umbilicales ${ }^{6}$. También se ha utilizado para mejorar o crear pezones en casos de pezón invertido o pobremente definido ${ }^{18}$.

Es un método eficaz por razones de peso. El avance centrípeto de la piel disminuye el diámetro de la herida, disminuyendo enormemente el área que tiene que sanar. La formación de hematomas es casi inexistente, ya que la abertura central sirve como portal para el drenaje ${ }^{2}$. Además, alcanza una adecuada hemostasia pues la sutura no sólo comprime los vasos en la dermis reticular, sino también los pequeños vasos presentes en la dermis papilar ${ }^{18}$.

Teóricamente, disminuye el potencial de infección 


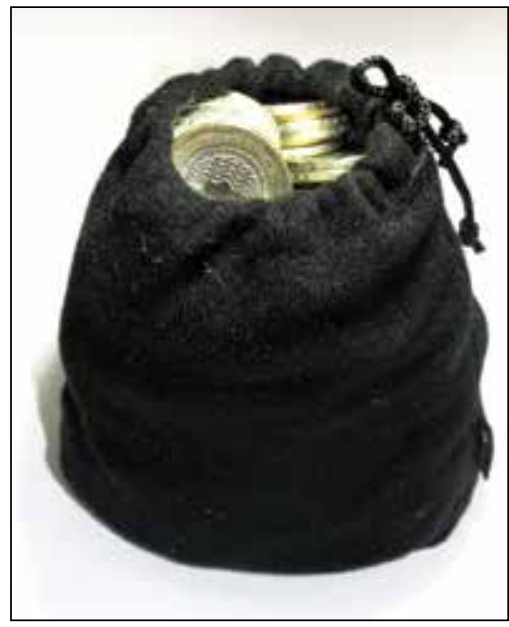

FIGURA 4. Bolsa portamonedas.

por el menor tamaño de la herida abierta y un menor tiempo de cicatrización en comparación con la cicatriz mayor en un cierre por segunda intención ${ }^{19}$. Con esta sutura ocurre una mínima distorsión de las estructuras adyacentes por razones geométricas, pues la tensión se aplica a lo largo de múltiples líneas radiales con un patrón de distribución concéntrica, en lugar de la tensión aplicada a lo largo de un eje perpendicular a la línea de la herida ${ }^{20}$. El calibre de las suturas empleadas varía de 6-o a 1-o, dependiendo del área de piel, pero usualmente se emplean las de mayores diámetros ${ }^{13}$. Nosotros empleamos calibres 3-0, 4-0 y 5-0.

En dermatología, los defectos quirúrgicos circulares secundarios a la cirugía micrográfica de Mohs, son especialmente adecuados para esta técnica ${ }^{12}$. Se ha utilizado en lesiones de cabeza y cuello para disminuir el diámetro del defecto quirúrgico y la posterior colocación de injertos cutáneos de espesor total, permitiendo la utilización de un injerto de menor tamaño, sin ejercer una mayor tensión ${ }^{21}$.

La sutura en bolsa de tabaco tiene varias ventajas:

1. Es una técnica simple, de fácil aprendizaje, rápida y de bajo costo.

2. Minimiza la cicatriz.

3. Es un cierre temporal útil en espera de un diagnóstico histológico definitivo.

4. Produce un excelente resultado cosmético y funcional en heridas seleccionadas, especialmente en áreas cóncavas y de piel laxa.
5. No se forman hematomas por la abertura central del defecto y por la hemostasia que produce la tensión sobre los bordes.

6. A diferencia de los injertos, evita la necesidad de una segunda herida quirúrgica del sitio donante y sus posibles efectos.

7. No tiene el riesgo de necrosis de los colgajos.

8. Es útil en piel atrófica.

9. Minimiza el número de pasos de aguja requeridos para el cierre en pacientes con enfermedades transmitidas mediante la sangre.

10. El retiro de la sutura es relativamente asintomático, pues sólo requiere el corte de un punto.

11. Es ideal en pacientes que desean continuar un estilo de vida activo durante el periodo posoperatorio.

12. Es adecuado para pacientes que reciben anticoagulantes o antiagregantes plaquetarios.

13. Es ideal para el manejo conservador de grandes heridas quirúrgicas que, de otra manera, requerirían injertos o colgajos para cerrar el defecto.

La principal desventaja de este método es la aceptación por el paciente, quien necesita ser preparado para la distorsión inicial, la tensión experimentada durante las primeras semanas y el largo tiempo que debe permanecer la sutura ( dos a cuatro semanas); sin embargo, la satisfacción del paciente con el resultado final es generalmente muy grande, especialmente cuando se trata de grandes defectos en la cara ${ }^{22,23}$. En este estudio el nivel de satisfacción del paciente con el procedimiento y el resultado final fue calificado entre bueno y excelente. En el cuero cabelludo, si se cierra con mucha tensión, puede presentar una tasa considerable de alopecia ${ }^{24}$.

En conclusión, es una técnica de fácil ejecución, que permite tratar heridas de gran tamaño ${ }^{25}$. El tiempo operatorio es ostensiblemente menor que el empleado en un cierre con injerto o colgajo. Puede practicarse con anestesia local en la mayoría de los pacientes, lo cual reduce los costos. Permite ver con anticipación las posibles recidivas tumorales locales, al no ejercer un efecto tapadera en la zona tratada ${ }^{3}$. En cirugía, el método ideal de reparación es aquel que minimiza la morbilidad y maximiza el resultado cosmético para el paciente ${ }^{26}$.

En principio, la cirugía dermatológica antepone, en su medida y circunstancia, el resultado curativo al cosmético, pero evidentemente también tiene que ofrecer unos resultados cosméticos aceptables por un imperativo social ${ }^{22}$. La cicatriz inicial es casi circular; posteriormente, luego de un período de semanas o meses, se orienta a lo largo de las líneas de tensión de la piel y es típicamente más corta que el defecto original. Las 


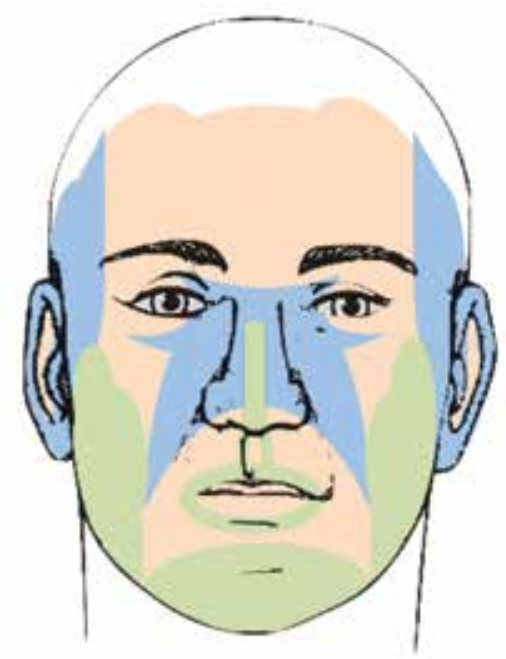

Excelente

Satisfactorio

Variable
FIGURA 5. Resultados cosméticos de la cicatrización por segunda intención según el sitio anatómico. heridas suelen curarse con excelentes resultados estéticos y funcionales, con cicatrices finales más cortas que la herida original ${ }^{13}$.

\section{Cicatrización por segunda intención}

El proceso de cicatrización se compone de una serie de eventos complicados y coordinados que tienen como objetivo la reconstrucción estructural y funcional del defecto $^{27}$. Se han identificado fases como la inflamatoria, la de proliferación y la de remodelado tisular, las cuales se separan con fines académicos, ya que en realidad funcionan simultáneamente para la reparación de la herida ${ }^{28}$.

En la era actual de técnicas de reconstrucción, elegantes y elaboradas, la curación por segunda intención a menudo se considera sólo como un último recurso o una modalidad reconstructiva no deseada ${ }^{26}$. Es una opción bien conocida en cirugía dermatológica y erróneamente se considera un método al que solamente recurren los cirujanos poco avanzados ${ }^{3}$.

La versatilidad de la curación espontánea se ha hecho evidente cuando se emplea para curar heridas que presentan complicaciones por infección, dehiscencia de sutura, necrosis del colgajo o pérdida del injerto ${ }^{18}$. Aunque hay disponibles varias opciones reconstructivas, en muchos casos, la opción más simple puede proveer los mejores resultados ${ }^{29}$.

En la extirpación de tumores mediante la cirugía de Mohs, por necesidad, a casi todas las heridas se les permite la cicatrización por segunda intención ${ }^{26}$. Esta cicatrización se produce por la contracción de la herida y su reepitelización. En la contracción, los bordes de la herida se unen, como resultado del movimiento centrípeto de la piel adyacente no lesionada hacia el centro del defecto. Es un fenómeno fundamental que produce una cicatriz mucho más pequeña que el defecto original ${ }^{29,30}$.

En circunstancias seleccionadas apropiadamente, la cicatrización por segunda intención ofrece un resultado funcional y cosmético igual o superior al alcanzado por medio de injertos y colgajos. Este eficiente y costo-efectivo método permite una óptima vigilancia de la herida, un bajo rango de complicaciones y evita complejos procedimientos en pacientes frágiles ${ }^{29}$. Puede ser el tratamiento de elección en pacientes ancianos, en los que de otra manera habría necesidad de un injerto de piel ${ }^{31,32}$.

Zitelli publicó en 1983 una completa revisión de los defectos faciales manejados con cicatrización por segunda intención, la cual se ha convertido en el artículo mas citado en la literatura dermatológica por los defensores de este tipo de cierre quirúrgico. La ubicación es un factor pronóstico importante del resultado estético, debido a las diferencias regionales en la contracción de las heridas $^{30}$. En general, las heridas en superficies cóncavas sanan con cicatrices casi imperceptibles, mientras que en las convexas sanan en forma más variable ${ }^{26,27}$.

Las heridas en las concavidades de la nariz (ala y pliegues nasolabiales), ojo (canto medial, párpado inferior), oreja (concha, fosa triangular) y la sien, sanan con excelentes resultados (FIGURA 5).

\section{Agradecimientos}

A mi maestra Lucía van den Enden, por su confianza e incondicional apoyo. 


\section{Referencias}

1. Greenbaum S, Radonich M. The purse-string closure. Dermatol Surg. 1996;22:1054-6.

2. Sabatino C, Greenbaum S. Modified purse-string closure for reconstruction of moderate/large surgical defects of the face. Dermatol Surg. 1999;25:215-20.

3. Vilalta A, Moreno G, Vidal L. Cierre de las heridas quirúrgicas mediante plicatura con aproximación forzada. Piel. 2001;16:133-40.

4. Cordoro K, Russell M. Minimally invasive options for cutaneous defects: Secondary intention healing, partial closure, and skin grafts. Facial Plast Surg Clin North Am. 2005;13:215-30.

5. Kawika N, Greenbaum S. Revisiting the purse-string closure: Some new methods and modifications. Dermatol Surg. 2003;29:672-6.

6. Artola J, Manrique P, Bilbao I. Biopsia mediante cierre en bolsa de tabaco presuturada de lesiones umbilicales neonatales. Piel. 2005;20:425-7.

7. Patel K, Telfer M, Southee R. A “round block" purse-string suture in facial reconstruction after operations for skin cancer surgery. Br J Oral Maxillofacial Surg. 2003;41:151-6.

8. Pipitone M, Gloster H. Repair of the alar groove with combination partial primary closure and second-intention healing. Dermatol Surg. 2005;31:608-9.

9. Cruz A, Campbell R, Perlis C, Dufresne R. Double purse-string closure for scalp and extremity wounds. Dermatol Surg. 2007;33:369-73.

10. Cohen P, Martinelli P, Schulze K, Nelson B. The purse-string suture revisited: A useful technique for the closure of cutaneous surgical wounds. Int J Dermatol. 2007;46:341-7.

11. Hoffman A, Lander J, Lee P. Modification of the purse-string closure for large defects of the extremities. Dermatol Surg. 2007;34:243-5.

12. Marquart J, Lawrence N. The purse-string lockdown. Dermatol Surg. 2009;35:853-5.

13. Zhu J, Wu X, Lu Z, Cai S, Zheng M. Purse-string suture for round and oval defects: A useful technique in dermatologic surgery. J Cutaneous Med Surg. 2012;16:11-7.

14. Romiti R, Randle H. Complete closure by purse-string suture after Mohs micrographic surgery on thin, sun-damaged skin. Dermatol Surg. 2002;28:1070-2.

15. Vujevich J, Obagi S. Repair of partial earlobe cleft using a "pursestring” repair. Dermatol Surg. 2006;32:969-71.

16. Adams B, Anwar J, Wrone D, Alam M. Techniques for cutaneous sutured closures: Variants and indications. Semin Cutan Med Surg. 2003;22:306-16.
17. Brady J, Grande D, Katz A. The purse-string suture in facial reconstruction. J Dermatol Surg OncoI. 1992;18:812-6.

18. Cohen P, Martinelli P, Schulze K, Nelson B. The cuticular purse string suture: A modified purse string suture for the partial closure of round postoperative wounds. Int J Dermatol. 2007;46:746-53.

19. Romaní J, Yébenes M. Repair of surgical defects of the nasal pyramid. Actas Dermosifiliogr. 2007;98:302-11.

20. Baraldini V, Coletti M, Cigognetti F, Vercellio G. Haemostatic squeezing and purse-string sutures: Optimizing surgical techniques for early excision of critical infantile haemangiomas optimizing surgical techniques for early excision of critical infantile haemangiomas. J Pediatr Surg . 2007;42:381-5.

21. Mather K, Harrington A, Montemarano A, Farley M. Surgical pearl: Purse string suture in the management of poorly delineated melanomas. J Am Acad Dermatol. 1998;38:99-101.

22. Tremolada C, Blandini D, Beretta M, Mascetti M. The "round block" purse-string suture: A simple method to close skin defects with minimal scarring. Plast Reconstr Surg. 1997;100:126-31.

23. Marquez T, Christoforidis D, Anasooya A, Madoff R, Rothenberger D. Wound infection following stoma takedown: Primary skin closure versus subcuticular purse-string suture. World J Surg. 2010;34:2877-82.

24. Kontos A, Ozog M. Use of purse-string suture technique in closure of flesh tunnel defects of the bilateral earlobes. Dermatol Surg. 2006;32:1070-1

25. Vlahovic A, Simic R, Kravijanac D. Circular excision and pursestring suture technique in the management of facial hemangiomas. Int J Pediatr Otorhinolaryngol. 2007;71:1311-5.

26. van der Eerden P, Lohuis P, Hart A, Mulder W, Vuyk H. Secondary intention healing after excision of non melanoma skin cancer of the head and neck. Plast Reconstr Surg. 2008;122:1747..

27. Rivera A, Spencer J. Clinical aspects of full-thickness wound healing. Clin Dermatol. 2007;25:39-48.

28. Benavides J. Reparación de heridas cutáneas, Rev Asoc Col Dermatol. 2008;16:29-35.

29. Neuhaus I, Yu S. Second-intention healing of nasal alar defects. Dermatol Surg. 2011; Vol.101-6.

30. Mott K, Clark D, Sue L. Regional Variation in wound contraction of Mohs surgery defects allowed to heal by second intention. Dermatol Surg. 2003;29:712-22.

31. Fife D, Alam M. Alternative techniques for reduction of scar length during staged excision. J Am Acad Dermatol. 2011;65:811-8.

32. Primary Care Dermatology Society. Skin Surgery Guidelines, 2007. Fecha de consulta: Oct.8 de 2.012; Disponible en: http://www. pcds.org.uk/images/downloads/skin_surgery_guidelines.pdf. 